\title{
Experimental analysis of reacting and non-reacting sprays
}

\author{
Lola Rousseau ${ }^{* 1}$, Mikael Orain ${ }^{2}$, Olivier Rouzaud ${ }^{1}$, Pascal Fede $^{3}$, Olivier Simonin ${ }^{3}$ \\ ${ }^{1}$ ONERA DMPE Centre Midi-Pyrénées, Toulouse, France \\ ${ }^{2}$ ONERA DMPE Centre Midi-Pyrénées, Fauga-Mauzac, France \\ ${ }^{3}$ Institut de Mécanique des Fluides de Toulouse (IMFT), Université de Toulouse, CNRS, \\ Toulouse, France \\ *Corresponding author email : lola.rousseau@onera.fr
}

\begin{abstract}
Introducing liquid fuel inside an aeronautical combustion chamber is associated with several complex phenomena like droplet dispersion by turbulence, spray evaporation and combustion. In order to study experimentally the spray behaviour, under non-reactive and reactive conditions, Mie scattering images provided by the PROMETHEE-LACOM test rig have been processed. However, the transition between a 3D flow in the combustion chamber to a 2D planar Mie scattering images leads to some errors. The present paper focuses on the evaluation of these errors and the influence on the droplet spatial distribution. Results show that the droplet distribution remains practically the same between the 3D $-2 D$ transition. Moreover, the restriction error decreases when droplet density number increases and the projection error increases when droplet density number increases.
\end{abstract}

\section{Keywords}

Spray, Inter-droplet distance, Preferential segregation, Evaporation, Combustion

\section{Introduction}

In aeroengines, fuel is injected as a liquid into the combustion chamber in order to improve combustion efficiency. This process involves two-phase flow turbulent combustion where many phenomena occur, such as atomization, droplet dispersion by turbulence or spray evaporation, and affect the combustion process. Some models were developed to attempt for describing more accurately spray evaporation and combustion including works of Chiu et al. [1], Kerstein et al. [2] or Sirignano [3]. In some of these theoretical models, distance parameter and droplet spatial distribution are necessary to describe and model the evaporation and the combustion of a spray. Usually, distance parameter is calculated according to the mean droplet density number and the droplet spatial distribution is fixed as a simple regular grid distribution, so the droplets are regularly arranged in the spray ([1], [4]). Experimentally, Vicentini [5], Rouzaud et al. [6] and Rousseau et al. [7] had studied the behaviour of a spray by means of the test rig PROMETHEE, which provides flow conditions close to those encountered inside aeroengine combustion chamber. They noticed that, for non-reactive and reactive conditions, that a linear relationship between the inverse square root of droplet number density in the spray and the mean inter-droplet distance exists (Fig. 1). Moreover, they observed that the experimental droplet spatial distribution, obtained from Mie scattering images, is close to the theoretical 2D uniformly randomly distribution, also called 2D Hertz-Chandrasekhar distribution.

However, the planar Mie scattering technique provides only two-dimensional images. Therefore, calculating the nearest-neighbour inter-droplet distance, or any distance, from the images implies necessarily some approximations since the transversal dimension is not taken into account. Vicentini [5] and Rouzaud et al. [6] have proposed to use three dimensional droplet fields to evaluate the errors committed on the distance when considering only two dimensional images. The same remark can be done for the spray spatial distribution.

Present work detailed in this paper follows on these suggestions. Two major axes are investigated. The first one is the evaluation of the errors committed on the nearest-neighbour inter-droplet distance throughout of the transition between three-dimensional to twodimensional fields. The second one is the influence of this transition on the preferential 
segregation characterization. To this end, the reminder of the paper comprises three sections. The numerical data used and the processing algorithm are first detailed. Results for a spray uniformly randomly distributed and for a spray with preferential segregation are presented and compared. Finally, the paper ends with a summary of the main findings and perspectives.
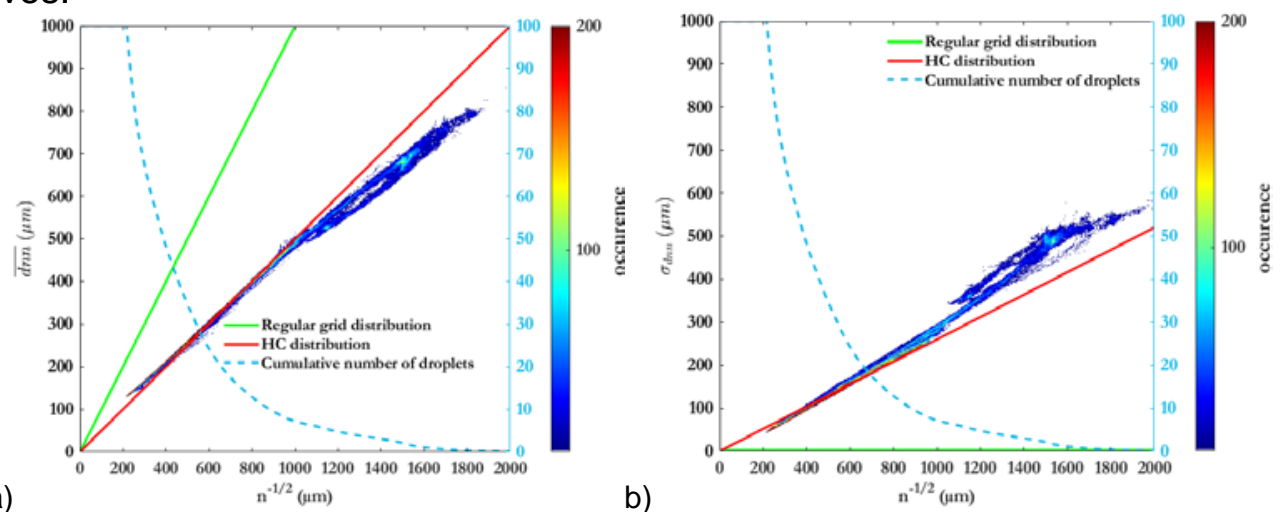

Figure 1. dnn - droplet number density diagram for reacting conditions. a) mean dnn b) standard deviation of dnn. Diagrams taken from [7]

\section{Material and Methods}

\section{Numerical database}

- Hertz-Chandrasekhar solution

A Monte-Carlo simulation tool has been developed in MATLABC to create computational volumes where droplets are uniformly randomly distributed in a 3D cube. The mean droplet number density $\left(\overline{n_{3 D}}\right)$, the number of droplet $\left(N_{d}\right)$ and the side length of the computational volume $\left(c_{\text {cube }}\right)$ can vary according to the desired configuration. The analysis makes also appear a mean number of droplets per unit surface or, in other words, a mean surface density number $\overline{n_{2 D}}$.

- Preferential segregation numerical data

To study the effects of the preferential segregation, six Direct Numerical Simulation coupled with Discrete Particle Simulation (DNS-DPS) [8] have been used, considering the same isotropic homogeneous turbulent gaseous flow but different solid particles density leading to different particle distribution. The computational domain is a cube with a size of $2 \pi \mathrm{m}$, containing 140367 spherical particles with a constant diameter of $15 \mathrm{~cm}$. The particle density number is around to $6 \times 10^{-4}$ droplet per $\mathrm{cm}^{3}$. To highlight the preferential segregation effect, the particle mass density varies from $12.5 \mathrm{~kg} . \mathrm{cm}^{-3}$ to $1250 \mathrm{~kg} . \mathrm{cm}^{-3}$. The Stokes particle number is between 0.33 to 14.42 and is defined as follow:

$$
\text { St }=\frac{\tau_{p}}{\tau_{k}}
$$

where $\tau_{p}$ is the particle relaxation time and $\tau_{k}$ the Kolmogorov time scale, equal to $0.3088 \mathrm{~s}$ in these simulations. Figure 2 shows slices of the droplet spatial distribution according to four different Stokes numbers. For Stokes numbers equal to 1.37 (b) and 5.06 (c), preferential segregation is clearly observed.
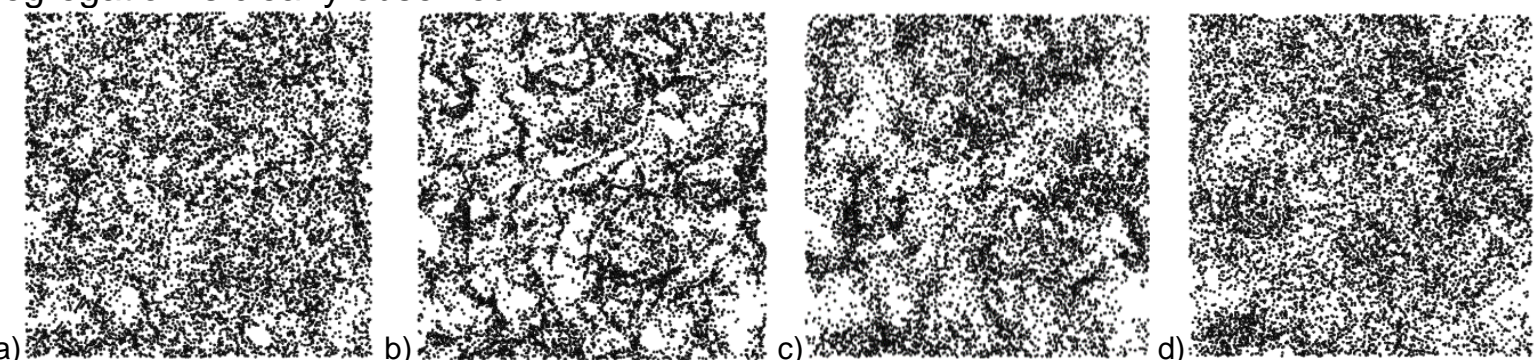

Figure 2. Visualizations of droplet spatial distribution ([2]) a) $\mathrm{St}=0.33 \mathrm{~b}$ ) $\mathrm{St}=1.37 \mathrm{c}$ ) $\mathrm{St}=5.06 \mathrm{~d}$ ) $\mathrm{St}=14.42$ 


\section{Data processing}

Mie scattering measurement technique provides two-dimensional images from the observation of a three-dimensional flow. In order to evaluate the influence of this transition between 3D to 2D for a better experimental Mie scattering images analysis, a numerical data processing has been developed to reproduce as precisely as possible the transition between a three-dimensional droplet field and the associated two-dimensional field. It is structured into two main steps: the first one is to divide the computational volume into a finite number of slices of thickness $e$ and calculate the mean and the standard deviation of the inter-droplet distance in these slices, the second one is used to project in a plan the droplets contained in the slices to have numerical images similar to the experimental ones.

Firstly, choosing a thickness $e$ for a slice and the surface density number $\overline{n_{2 D}}$, the volume droplet density number $\overline{n_{3 D}}$ is:

$$
\overline{n_{3 D}}=\frac{\overline{n_{2 D}}}{e}
$$

The side length of the computational volume $c_{\text {cube }}$ and the number of slices $N_{s}$ are equal to:

$$
\left\{\begin{array}{c}
c_{\text {cube }}=\left(\frac{N_{d}}{\overline{n_{3 D}}}\right)^{\frac{1}{3}} \\
N_{s}=\frac{c_{\text {cube }}}{e}
\end{array}\right.
$$

In the whole volume, the value $d n n_{3 D}$ represents the smallest Euclidian distance between a droplet " $d$ " and its nearest-neighbor " $d$ " as:

$$
d n n_{3 D}^{d}=\sqrt{\left(x_{d}-x_{d^{*}}\right)^{2}+\left(y_{d}-y_{d^{*}}\right)^{2}+\left(z_{d}-z_{d^{*}}\right)^{2}}
$$

while the mean nearest-neighbour inter-droplet distance is calculated as:

$$
\overline{d n n_{3 D}}=\frac{1}{N_{d}} \sum_{d=1}^{N_{d}} d n n_{3 D}^{d}
$$

Similarly to the volume, in a slice "s", the value $d n n_{3 D}^{d, s}$ is the smallest Euclidian distance between a droplet " $d$ " and its nearest-neighbour " $d$ " contained is the slice and the mean value on a slice becomes:

$$
\overline{d n n_{3 D}^{\text {restriction }}}=\frac{\sum_{s=1}^{N_{s}}\left(\sum_{d=1}^{N_{d}^{s}} d n n_{3 D}^{d, s}\right)}{\sum_{s=1}^{N_{S}} N_{d}^{S}}
$$

where $N_{d}^{S}$ is the number of droplets in the slice "s". For both volume and slice, the standard deviation is calculated in the same way.

Secondly, for the orthogonal projection, a droplet " $d$ " contained in the slice " $s$ " with the coordinates $(x, y, z)$, and its projection in a plan " $i$ " (like an image) according to the $\vec{x}$ axe is $(y, z)$. In this way, the nearest-neighbour inter-droplet distance calculated in the image, its mean value in the volume and the associated mean surface droplet density number are calculated as:

$$
\left\{\begin{array}{c}
d n n_{2 D}^{d, i}=\sqrt{\left(y_{d}-y_{d^{*}}\right)^{2}+\left(z_{d}-z_{d^{*}}\right)^{2}} \\
\overline{d n n_{2 D}^{\text {projection }}}=\frac{\sum_{i=1}^{N_{i}}\left(\sum_{d=1}^{N_{d}^{i}} d n n_{2 D}^{d, i}\right)}{\sum_{i=1}^{N_{i}} N_{d}^{i}} \\
\overline{n_{2 D}^{\text {projection }}}=\frac{\sum_{i=1}^{N_{i}} N_{d}^{i}}{N_{i} * S_{i}}
\end{array}\right.
$$

where $N_{d}^{i}$ is the number of droplet in an projected image, $N_{i}=N_{S}$ and $S_{i}$ the surface of the image " $i$ ". The standard deviation is calculated in the same way. 
Theoretical laws

In the two-dimensional case, linear relationships between the inverse square root of the mean droplet density number and the mean nearest-neighbour inter-droplet distance and its standard deviation have been previously observed using the experimental data in both nonreactive and reactive cases [6] [7] and are defined as:

$$
\left\{\begin{array}{l}
\overline{d n n_{2 D}}=\alpha_{2 D} *{\overline{n_{2 D}}}^{-\frac{1}{2}} \\
\sigma_{d n n_{2 D}}=\beta_{2 D} * \bar{n}_{2 D}-\frac{1}{2}
\end{array}\right.
$$

where $\alpha_{2 D}$ and $\beta_{2 D}$ are the corresponding dimensionless mean and standard deviation of the nearest-neighbour inter-droplet distance distribution. In the case of the simple grid distribution where droplets are regularly arranged, $\alpha_{2 D}$ equals 1 and $\beta_{2 D}$ equals 0 . In the case of the 2D Hertz-Chandrasekhar distribution, $\alpha_{2 D}$ is equal to 0.50 and $\beta_{2 D}$ is equal to 0.26 .

In the same way, for the three-dimensional case, the linear relationship is defined as:

$$
\left\{\begin{array}{l}
\overline{d n n_{3 D}}=\alpha_{3 D} * \overline{n_{3 D}}-\frac{1}{3} \\
\sigma_{d n n_{3 D}}=\beta_{3 D} * \overline{n_{3 D}}-\frac{1}{3}
\end{array}\right.
$$

In the case of the simple grid distribution where droplets are regularly arranged, $\alpha_{3 D}$ equals 1 and $\beta_{3 D}$ equals 0 . In the case of the 3D Hertz-Chandrasekhar distribution, $\alpha_{3 D}$ is equal to 0.5539 and $\beta_{3 D}$ is equal to 0.2013 .

\section{Results and Discussion}

3D study for theoretical Hertz-Chandrasekhar distribution and DNS-DPS simulations

In the case of the Hertz-Chandrasekhar distribution, the evolution of the $\overline{d n n_{3 D}}$ and the $\sigma_{d n n_{3 D}}$ calculated for different volumetric droplet density number are shown in the figure 3. While keeping the number of droplets fixed (140367), the density numbers vary between $6 \mathrm{x}$ $10^{-5}$ and 45000 droplet per $\mathrm{cm}^{3}$, and are representative of the experimental data and of the DNS/DPS simulations density number values. The red line corresponds to the 3D theoretical Hertz-Chandrasekhar distribution and the dashed black line to the results of the Monte-Carlo simulations. As expected, results of Monte-Carlo simulations are very close to the theoretical 3D Hertz-Chandrasekhar distribution. Therefore, the Monte-Carlo code is validated and the retained number of droplets is sufficient to perform a statistical sampling.
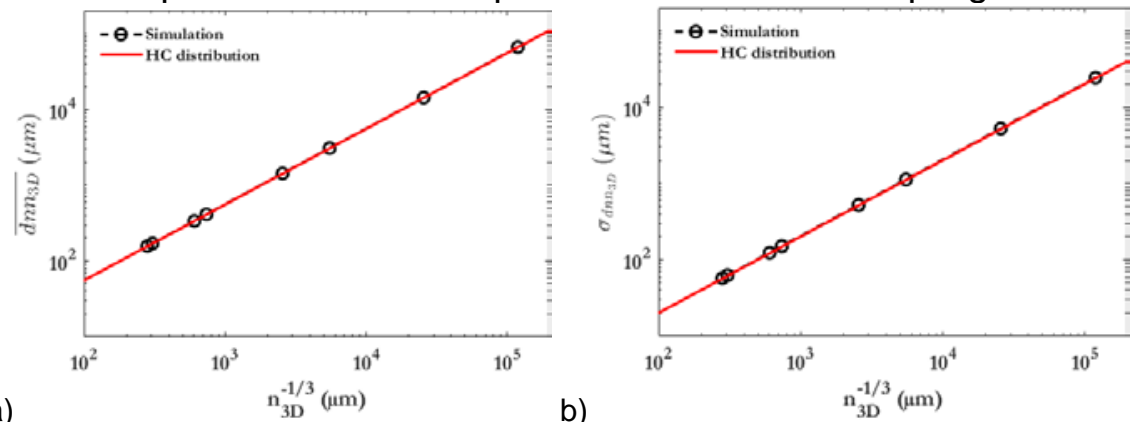

Figure 3. Evolution of a) mean dnn and b) standard deviation for Monte-Carlo simulations

In the case of preferential segregation, Vicentini [5] and Boutsikakis [9] have observed that the dimensionless mean $(\alpha)$ and the dimensionless standard deviation $(\beta)$ highlight its presence. Figure 4 shows the evolution of $\alpha_{3 D}$ and $\beta_{3 D}$ according to the Stokes number. The red line corresponds to the dimensionless variables for the theoretical 3D HertzChandrasekhar distribution and the dashed black line corresponds to the results of DNSDPS simulations. Two regions can be observed. In the first part corresponding to a Stokes number lower than $1.4, \alpha_{3 D}$ decreases and $\beta_{3 D}$ increases when the Stokes number increases, and they both deviate from their theoretical values. In the second region, for 
Stokes number values higher than $1.4, \alpha_{3 D}$ decreases and $\beta_{3 D}$ increases when the Stokes number increases, and both tend toward the theoretical value for high Stokes values. This observation is consistent with the aforementioned results ([5], [9]). When Stokes number is very small, droplets behave as fluid particle and are dispersed everywhere, obeying the 3D Hertz-Chandrasekhar distribution. With an increasing Stokes number, preferential segregation appears with a clear variation of $\alpha_{3 D}$ and $\beta_{3 D}$ coefficients. Finally, for very inertial droplets, the droplet spatial distribution tends again to be close to 3D HertzChandrasekhar distribution.
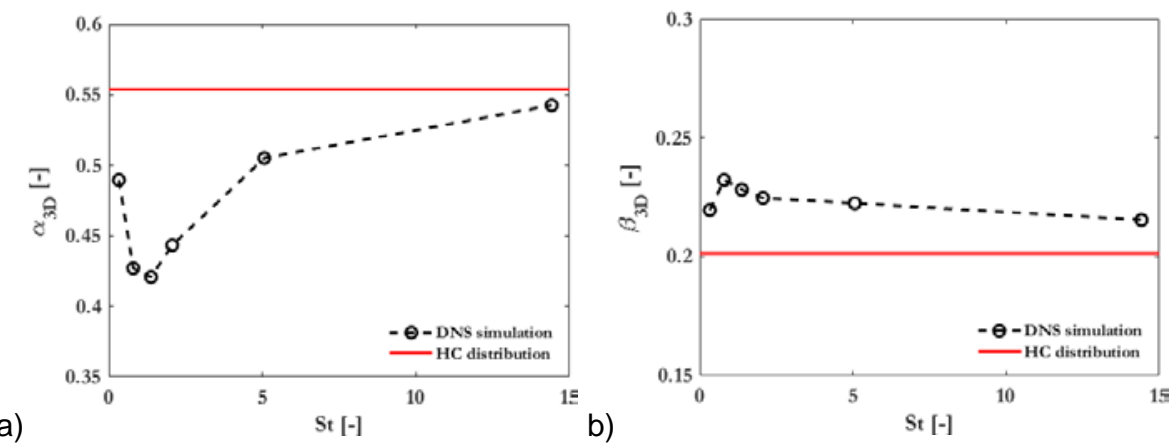

Figure 4. Evolution of a) dimensionless mean $(\alpha)$ b) dimensionless standard deviation $(\beta)$ according to the Stokes number for DNS simulations

\section{Study of 3D - 2D transition effect on theoretical Hertz-Chandrasekhar distribution}

The transition between a 3D droplet field distribution and its associated 2D image is made of two steps. The first one corresponds to the transition from the complete 3D volume to the slice volume corresponding to the laser sheet. Therefore, it is possible that some droplets contained in the slice have their nearest-neighbour droplet outside of this slice. For these droplets, the nearest-neighbour distance calculated with the droplets only contained inside the slice is no longer correct. The name "3D restriction effect" is used to indicate this effect. The second step corresponds to the projection of the droplets contained in the slice to a 2D surface. Since the distance between the droplets in the slice is most of time greater than the distance between the projected droplets in the 2D image, the nearest-neighbour inter-droplet distance is modified. The name "projection effect" is used to indicate this effect. Thanks to the Monte-Carlo solver, it is possible to quantify independently the influence of these effects on the droplet spatial distribution. In order to do so, the number of droplets contained in the computational volume is kept constant and equal to 140367 according to the previous result. Besides, we also prescribe the surface droplet density numbers. They are fixed to [250 450 3500 4500] droplets per $\mathrm{cm}^{2}$ and correspond to the extremal experimental values used for the non-reactive and reactive cases [7]. Finally, the thickness of the slice (laser sheet) is equal to $1 \mathrm{~mm}$ which represents around 2 at 6 times larger than the $\overline{d n n_{3 D}}$. Note that the side length of the volume varies from $14 \mathrm{~mm}$ to $38 \mathrm{~mm}$.

- 3D restriction effect

Figure 5 shows the evolution of the $\overline{d n n_{3 D}}$ value (Fig. 5 a) and the $\sigma_{d n n_{3 D}}$ value (Fig. $5 \mathrm{~b}$ ) according to the mean volume droplet number density $\overline{n_{3 D}}$. The red line corresponds to the theoretical Hertz-Chandrasekhar distribution. The dashed black line represents the case where the dnn of the droplets contained in a slice is calculated with the correct dnn values ("simulation without restriction"). The dotted blue line corresponds to the case where the dnn of each droplet in the slice is calculated only with the droplets inside the slice ("simulation with restriction").

As expected, for the mean and standard deviation values, the simulation without restriction is equivalent to the theoretical value. In the case with the restriction effect, the mean and standard deviation values are always overestimated according to the theoretical 3D HertzChandrasekhar distribution. However, the error committed decreases when the droplet density number increases which can be explained by the fact that the distance between the 
droplets decreases when the density increases, thus there is less probability for a droplet to have its nearest-neighbour outside the slice.
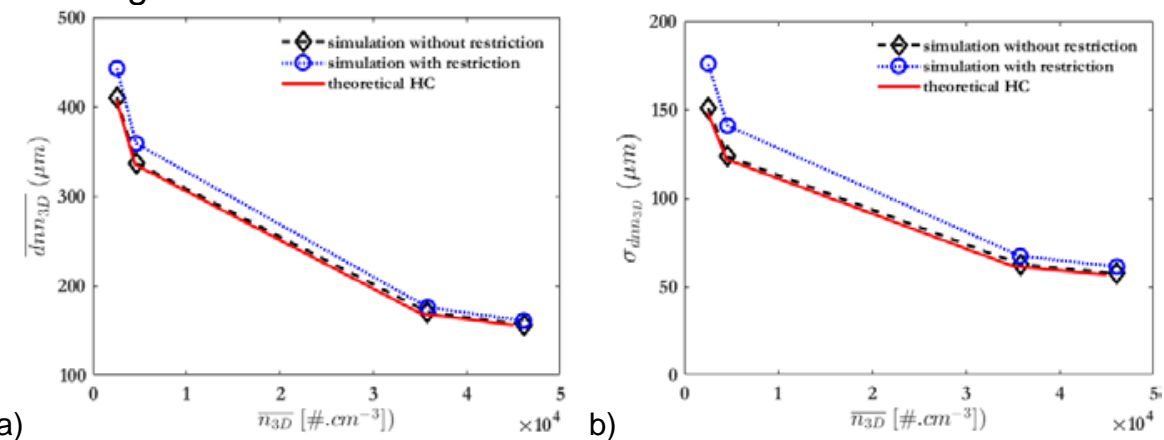

Figure 5. Comparison of a) $\overline{d n n_{3 D}}$ and b) $\sigma_{d n n_{3 D}}$ according the presence or not of 3D restriction effect for Monte-Carlo simulations $(e=1 \mathrm{~mm})$

- 2D projection effect

Figure 6 shows the behaviour of the dimensionless mean (Fig. 6 a) and standard deviation (Fig. 6 b) according to the surface droplet density number after the projection step. The red line represents the theoretical value for a 2D Hertz-Chandrasekhar distribution. The dashed black line corresponds to the projected results of the Monte-Carlo simulations.
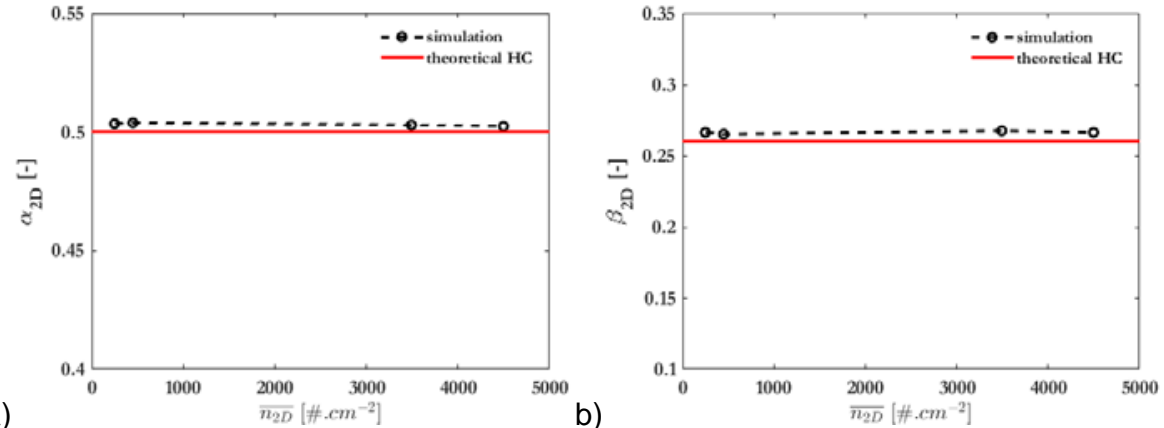

Figure 6. Estimation of a) $\alpha_{2 D}$ and b) $\beta_{2 D}$ after projection step for Monte-Carlo simulations $(\mathrm{e}=1 \mathrm{~mm})$

For each surface droplet density number, $\alpha_{2 D}^{\text {projection }}$ and $\beta_{2 D}^{\text {projection }}$ values are close to their theoretical values. It demonstrates that the projection of droplets uniformly randomly distributed in a volume or in a slice leads to a droplet spatial distribution close to the 2D Hertz-Chandrasekhar distribution. Conversely, it may be assumed that if a 2D droplet spatial distribution is close to the theoretical 2D Hertz-Chandrasekhar distribution, the 3D droplet spatial distribution should be close to the theoretical 3D Hertz-Chandrasekhar distribution.

- Estimation of the error throughout the 3D - 2D transition

Table 1 sums up the relative errors on the mean dnn and standard deviation of dnn values calculated respectively to $\overline{d n n_{3 D}}$ and $\sigma_{d n n_{3 D}}$.

Table 1. Relative error on mean dnn and standard deviation values calculated according to $\overline{d n n_{3 D}}$ and $\sigma_{d n n_{3 D}}$

\begin{tabular}{|c|c|c|c|c|c|c|}
\hline$\overline{n_{2 D}}\left[\mathrm{~cm}^{-3}\right]$ & $\frac{\text { Error for }}{\text { dnn }_{3 D}^{\text {restriction }}}$ & $\frac{\text { Error for }}{d n n_{2 D}^{H C}}$ & $\frac{\text { Error for }}{\text { dnn }_{2 D}^{\text {projection }}}$ & $\begin{array}{l}\text { Error for } \\
\sigma_{d n_{3 D}}^{\text {projection }}\end{array}$ & $\begin{array}{c}\text { Error for } \\
\sigma_{d n n_{2 D}}^{H C}\end{array}$ & $\begin{array}{l}\text { Error for } \\
\sigma_{d n n_{2 D}}^{\text {projection }}\end{array}$ \\
\hline 250 & $9 \%$ & $22 \%$ & $22 \%$ & $1.9 \%$ & $10 \%$ & $14 \%$ \\
\hline 450 & $7 \%$ & $30 \%$ & $29 \%$ & $1.6 \%$ & $0.5 \%$ & $2 \%$ \\
\hline 3500 & $4 \%$ & $50 \%$ & $50 \%$ & $1.9 \%$ & $29 \%$ & $26 \%$ \\
\hline 4500 & $3 \%$ & $52 \%$ & $52 \%$ & $1.4 \%$ & $31 \%$ & $30 \%$ \\
\hline
\end{tabular}

Several comments can be done. Firstly, the 3D restriction error committed when dividing the computational volume into slices of thickness $1 \mathrm{~mm}$ is less than $10 \%$ for the mean dnn and less than $2 \%$ for the standard deviation. Moreover, the largest the droplet density number is, the more the error is reduced. Secondly, the error associated with the projection step is 
close to the one found when using a 2D Hertz-Chandrasekhar distribution. It means that the error on the dnn calculated from the projection of droplets uniformly randomly distributed in a volume or a slice is close to the error on the dnn calculated for droplets which are distributed according to the 2D Hertz-Chandrasekhar distribution. This observation corroborates the fact that the projection of a 3D Hertz-Chandrasekhar distribution practically gives a 2D HertzChandrasekhar. Thirdly, for high droplet density number, the error on the mean dnn value is mostly due to the projection step whereas, for low droplet number density, the error is more well-balanced between the restriction and the projection steps.

\section{Study of transition between 3D - 2D for flow with preferential segregation}

The slice thickness of $1 \mathrm{~mm}$ is not suitable of the DNS-DPS configuration due to the side length of the computational volume $(6.28 \mathrm{~m})$. For this reason, it is necessary to define an appropriate thickness for this study in order to evaluate later the influence of the projection effect on the dimensionless mean dnn and standard deviation values in the presence of preferential segregation.

- Slice thickness selection

Figure 7 shows the evolution of the dimensionless mean dnn (Fig. 7 a) and standard deviation (Fig. $7 \mathrm{~b}$ ) according to the thickness of the slice in the case of the Monte-Carlo simulation for the same conditions than the DNS-DPS simulation. The red line represents the theoretical dimensionless values for a 2D Hertz-Chandrasekhar distribution. The dashed black line corresponds to the results of the Monte-Carlo simulation. In this figure, it can be observed that the $\alpha_{2 D}^{\text {projection }}$ and $\beta_{2 D}^{\text {projection }}$ seem to tend towards the 2D theoretical values for a thickness larger than $400 \mathrm{~mm}$.
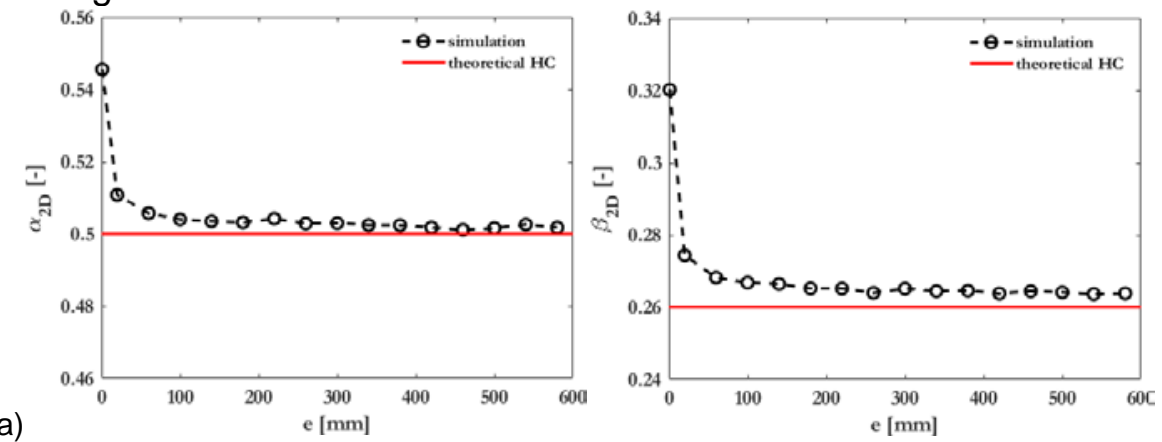

Figure 7. Evolution of a) $\alpha_{2 D}$ and b) $\beta_{2 D}$ for a Monte-Carlo simulation with a volume droplet density number of $6 \times 10^{-4}$ droplet per $\mathrm{cm}^{3}$

In order to see the influence of the slice thickness in the preferential segregation case, three thickness values were tested: 125,400 and $600 \mathrm{~mm}$. The first value comes from the experimental work ([5], [6], [7]) and corresponds to a ratio between the laser sheet thickness and the image dimensions around 0.02 . The $400 \mathrm{~mm}$ and $600 \mathrm{~mm}$ values are based on the preliminary analysis with the Monte-Carlo simulation.

- Influence on 2D projection effect

Figure 8 represents the evolution of the dimensionless mean dnn (Fig. 8 a) and standard deviation of dnn (Fig. 8 b) calculated after projection according to the Stokes number. The evolutions of $\alpha_{2 D}^{\text {projection }}$ and $\beta_{2 D}^{\text {projection }}$ are similar to those observed in the whole volume without restriction (see Fig. 4). This implies that the projection effect does not preclude the observation of the preferential segregation effect on the dimensionless values. Hence, these values are possibly, as in the three-dimensional case, a tool to detect the presence of preferential segregation in a two-dimensional image. Moreover, as for the previous part with a uniformly randomly distribution of droplets, if the droplet spatial distribution in an image shows signs of preferential segregation then the three-dimensional flow visualized and represented by this image should be close of this type of distribution. It can be also observed 
that, for St comprised between 0.8 and $5, \alpha_{2 D}$ value increases when slide thickness increases. This effect is less significant for $\beta_{2 D}$ value.

b)
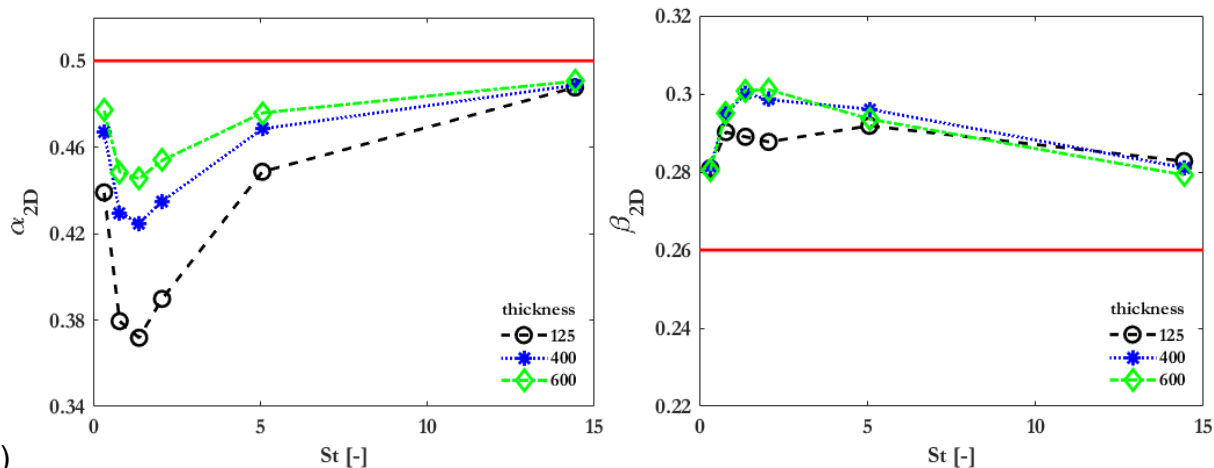

Figure 8. Evolution of $\alpha_{2 D}$ and $\beta_{2 D}$ according of the slice thickness (preferential segregation case)

\section{Conclusions}

The Mie scattering measurement technique implemented on the PROMETHEE experimental test rig provides two-dimensional images when a three-dimensional flow is observed, which induced some bias. In this paper, results of numerical simulations obtained from a MonteCarlo code and from DNS-DPS of isotropic uniformly turbulent two phase flow simulations have been processed in order to evaluate these biases. It was found that the 3D restriction bias generates a maximal error of $10 \%$ on the mean and standard deviation of dnn but it decreases when the droplet number density increases. Moreover, for both uniformly randomly spatial distribution and preferential segregation distribution, the distributions are similar in two and three dimensions. Further work will be done to extend theses numerical results on the experimental data and to extend the research about the influence between laser sheet thickness, length scale turbulence and droplets clusters/voids size.

\section{Acknowledgments}

The work has been realized thanks to the partial financial support of the "Région Occitanie". The numerical simulations were performed on the supercomputer Olympe (CALMIP) using time available under project P0111 and on the supercomputer Occigen (CINES) under the allocation A0082B0612.

\section{Nomenclature}

$\bar{n}:$ droplet density number

$\overline{d n n}$ : mean nearest-neighbour inter-droplet distance

$\sigma_{d n n}:$ standard deviation of nearest-neighbour inter-droplet distance

$\alpha$ : dimensionless mean nearest-neighbour inter-droplet distance

$\beta$ : dimensionless standard deviation of nearest-neighbour inter-droplet distance

\section{References}

[1] Chiu H.H., Liu TM, 1977, Combustion Flame, Vol. 17, pp 127 - 142

[2] Kerstein A.R., Law C.K., 1982, $19^{\text {th }}$ Symp. On Combustion, The Comb. Institute

[3] Sirignano W.A., 2014, Progress in Energy and Combustion Science, Vol. 42, pp $54-86$

[4] Paulhiac D., 2015, PhD Thesis, INP Toulouse.

[5] Vicentini M., 2016, PhD Thesis, ISAE.

[6] Rouzaud O., Vicentini M., Lecourt R., Bodoc V. Simonin O., Fede P., 2021, Atomization and Sprays, to be published.

[7] Rousseau L., Lempereur C., Orain M., Rouzaud O., Simonin O., 2021, Experiments In Fluids, 62, 26.

[8] Fede P., Simonin O., Villedieu P., 2015, International Journal of Multiphase Flow, Vol. 74, pp. $165-183$.

[9] Boutsikakis A., 2020, PhD Thesis, IMFT. 